\title{
New developments in phase contrast transmission electron microscopy with electrostatic phase plate
}

\author{
D. Alloyeau*, W.K. Hsieh*, \\ N.P. Balsara ${ }^{* * * *}$ and C. Kisielowski ${ }^{*}$ \\ E.H. Anderson ${ }^{* *}$, \\ G. Benner ${ }^{* * *}$, \\ E.D. \\ Gomez ${ }^{* * * *}$, \\ ${ }^{*}$ National Center for Electron Microscopy, Lawrence Berkeley National Lab, CA 94720 \\ ${ }^{* *}$ Center for X-ray Optics, Lawrence Berkeley National Lab, CA 94720 \\ ${ }_{* * * *}^{* *}$ Carl Zeiss SMT AG Company, Germany \\ ${ }^{* * * * *}$ Department of Chemical Engineering, University of California, Berkeley, CA 94720
}

Soft materials are often composed of low atomic number elements forming large molecules, which generate only poor contrast in a conventional Transmission Electron Microscope (TEM). Usually, phase contrast of such materials is generated by deliberately defocusing the objective lens in order to convert the phase modulation into an intensity modulation in a suitable range of spatial frequencies. However, this simple approach of the weak phase objects problems induced several drawbacks (i.e. lowering the microscope resolution and reducing the object outline visibility). Therefore, it is challenging to improve the imaging of soft materials and particularly if you are concerning by soft / hard materials interface.

Using two levels electron beam lithography, vapor phase deposition techniques and FIB etching, we have fabricated an electrostatic phase plate originally proposed by Boersch [1] for in-focus imaging of weak phase object (Fig. 1). This device, based on an electrostatic Einzel lens consists of a ring electrode, shielded by grounded electrodes. Inserted in the Back Focal Plan (BFP) of a TEM, it permits to apply a uniform phase shift to the unscatterred beam traveling through the interior of ring, without interfered with the scattered beam (Fig 1c) [2]. Since the cut-off frequency is a determinant parameter in the electrostatic phase plate purpose, the outer dimension of the ring electrode has been minimized until $0.7 \mu \mathrm{m}$, while keeping a large aperture size $(50 \mu \mathrm{m}$, Fig 1b). This device has been installed in a Zeiss LIBRA 200FE microscope equipped with a high tilt objective lens (focal length of $3 \mathrm{~mm}$ ). These technical improvements lead to the smallest cut-off frequency $\left(1 / 10.8 \mathrm{~nm}^{-1}\right)$ ever obtained with a Boersch phase plate, without affecting the high resolution ability of the microscope (0.28 $\mathrm{nm}$ at Scherzer focus).

First tests of the phase plate performances have been realized on Multi-Walled Carbon Nanotubes (MWCN) with a diameter bellow $10 \mathrm{~nm}$, deposited on holey amorphous carbon film. A piezo-driven manipulator finely controls the position of the device in the BFP thereby avoiding interference effects. A quantitative study of the phase shift induced by the phase plate has been realized by analyzing the influence of the electrode potential on the power spectra of the images (Fig. 2a). By finely comparing experimental and simulated Thon rings, we have shown that the phase shift is linear with the electrode potential (Fig. 2b).

Then, we have brought to light for the first time the influence of a Boersch phase plate on the contrast of weak phase objects. The figure 3 shows that by introducing a $\pi / 2$ phase shift we greatly increase the contrast of MWNT while remaining at 0 focus. This phase contrast enhanced image (fig. $3 \mathrm{~b}$ ) has a signal to noise ratio five time higher classical TEM picture (Fig 3c). Such a contrast gain is in good agreement with MWCN image calculated with an additional positive $\pi / 2$ phase shift (inset figures $3 b$ ).

Thus, electrostatic phase plate can be used to improve in focus imaging of poor contrast objects. Further works are now in progress to improve the imaging of soft materials (vesicles, proteins, polymers). In addition, we proved that $0.32 \mathrm{~nm}$ lattice distance can be resolved in spite of the phase plate insertion in the back focal plan. Therefore, the fine control on the applied phase shift demonstrated in this work, opens the way to wave function reconstruction from through phase series in high resolution mode. 
[1] H. Z. Boersch, Naturforsch. 2A (1947) 615

[2] T. Matsumoto et al., Ultramicroscopy 63 (1996), 5-10
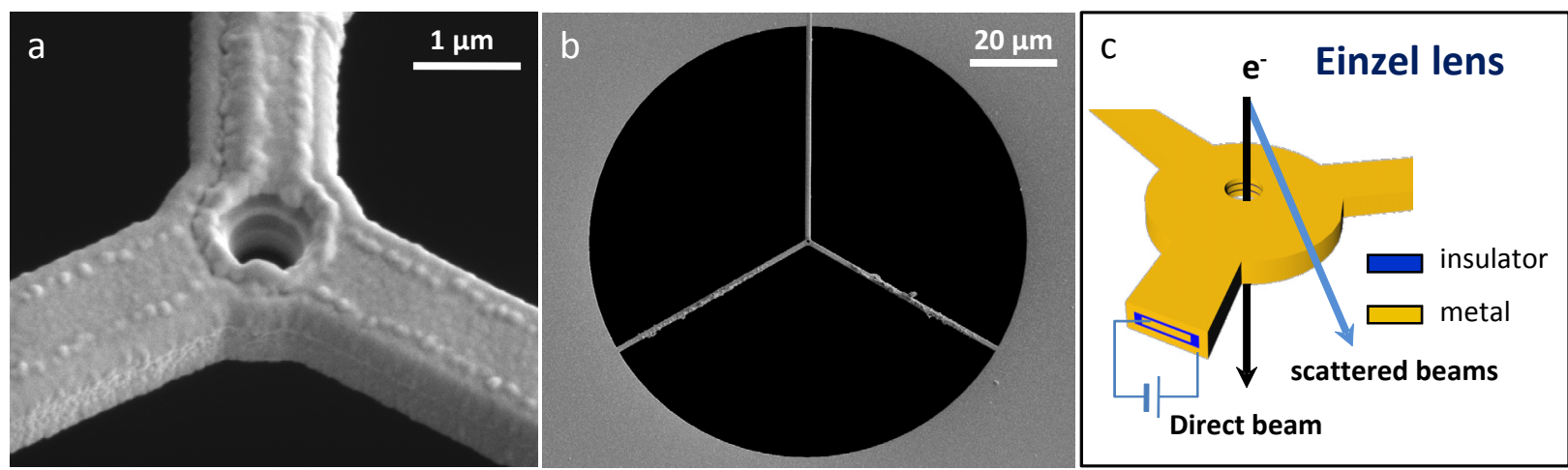

Fig. 1: (a) and (b) SEM images of a $1 \mu$ m outer-radius phase plate. (c) Basic principle of a phase plate.
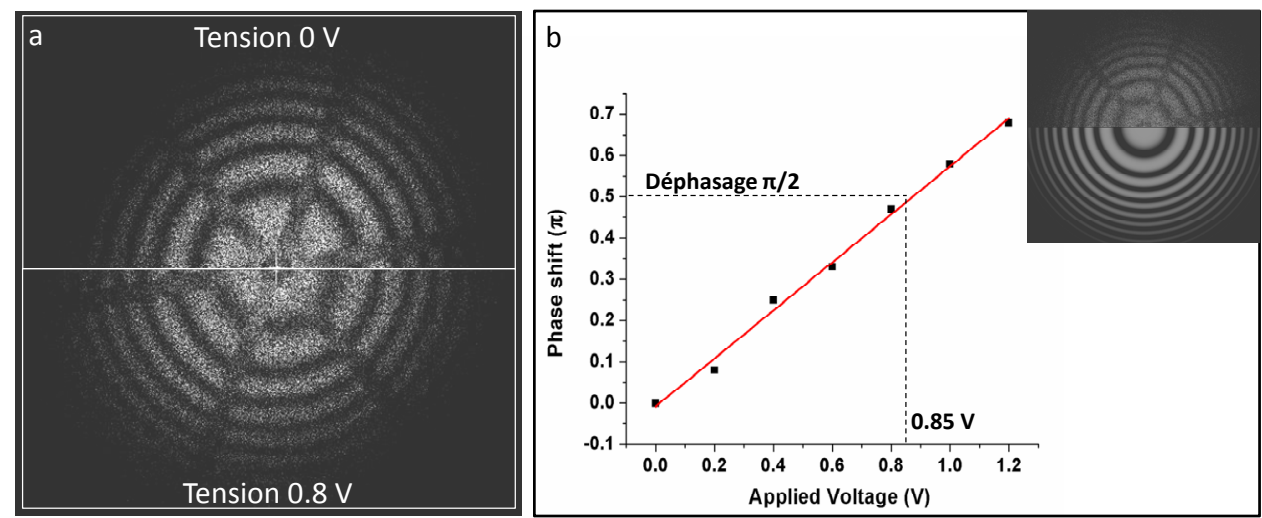

Fig. 2: (a) Composed power spectra from images acquired with an applied voltage of $0 \mathrm{v}$ (upper half) and $0.8 \mathrm{~V}$ (lower half) (b) Linear relationship between the phase shift and the applied voltage deduced from comparison with simulated thon rings (inset).
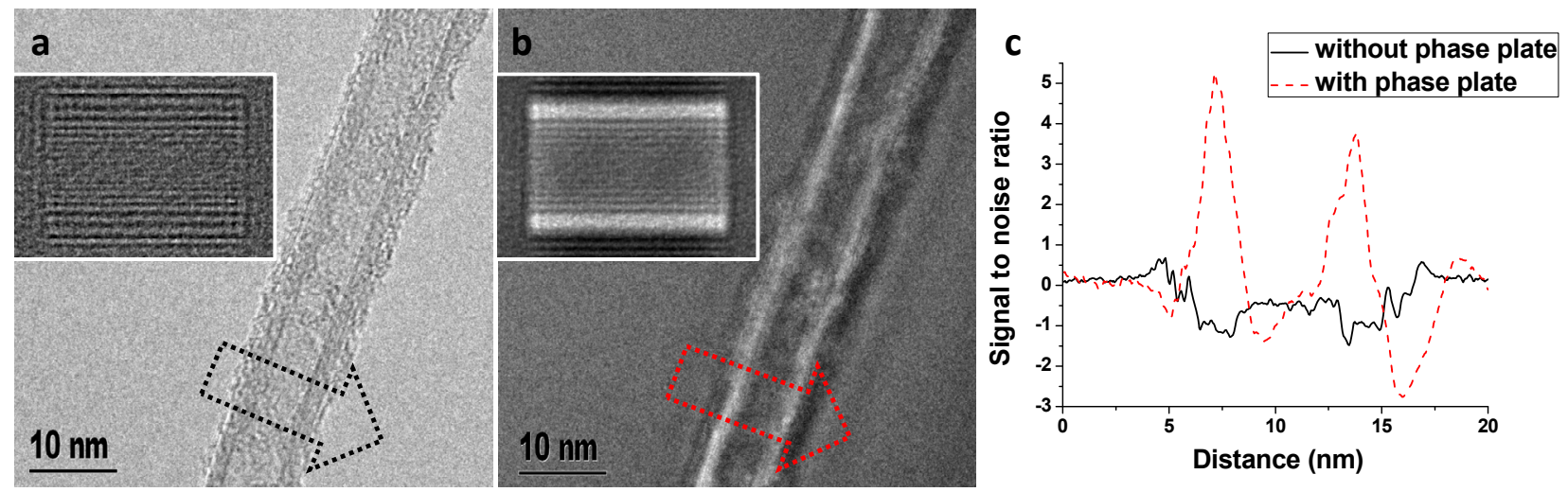

Fig. 3: TEM Images of a Multi Walled Carbon Nanotube (MWCT) with the corresponding simulated images (insets): (a) without phase plate close to zero focus, (b) with a phase plate applying a $\pi / 2$ phase shift close to zero focus. (c) Signal to noise ratio profile measured across the MWCT as indicated on the images $a$ and $b$ (arrows). 